\title{
Tunable $\mathrm{CO}_{2}$ Capture Effect in Visible Light Responsive MOF@MRs.
}

Richelle Lyndon $\uparrow$, Kristina Konstas $\ddagger$, Aaron. W. Thornton $\ddagger$, Aaron J. Seeber $\$$, Bradley P. Ladewig $\dagger$ and Matthew R. Hill $\$$

\section{SUPPORTING INFORMATION}

Table of Contents

Experimental Procedures

S1

Thermographic Analyses

S2

X-Ray Powder Diffraction

$\mathrm{S}_{3}$

Photoresponse Characterisation

$\mathrm{S}_{4}$

Light Responsive Control Experiments

$\mathrm{S}_{5}$

Light Responsive Gas Adsorption Experimental Setup'

S6 


\section{Section 1: Experimental Procedures S1}

The loading concentration (wt. \%) was calculated using the weight of MR in the mixture $\div$ the total mass of MR and MOF in the mixture $\times 100$. All materials were weighed on a 4 decimal place balance.

\section{S1.1 Synthesis of Mg-MOF-74}

$\mathrm{Mg}\left(\mathrm{NO}_{3}\right)_{2.6 \mathrm{H}} \mathrm{O}$ (1.96 g, $7.7 \mathrm{mmol}$ ), 2,5-dihydroxyterepthalic acid (o.47 g, $2.4 \mathrm{mmol}$ ) were dissolved in a solvent mixture of DMF (175 mL), EtOH (12.4 mL) and distilled water $(12.6 \mathrm{~mL})$ in a Teflon capped vial. The mixture was heated to $125{ }^{\circ} \mathrm{C}$ and the temperature was held for $20 \mathrm{~h}$, followed by cooling the mixture to room temperature. The yellow powder was then solvent exchanged in DMF for 2 days, followed by $\mathrm{MeOH}$ twice a day for five days. The dried sample was then activated at $200{ }^{\circ} \mathrm{C}$ for 5 hours at a rate of $1^{\circ} \mathrm{C} / \mathrm{min}$ under vacuum overnight.

\section{S1.2 Synthesis of Mg-MOF-74@MR}

Mg-MOF-74 (o.17 g) and methyl red (MR) (0.16 g) were mixed in DCM (5 mL) and the mixture was sonicated for 15 minutes and left overnight. The mixture was filtered and thoroughly washed with DCM to remove excess MR. Mg-MOF-74@MR was heated under vacuum at $150{ }^{\circ} \mathrm{C}$ overnight at a rate of $1{ }^{\circ} \mathrm{C} / \mathrm{min}$ to ensure solvent removal. Due to the rigidity of the framework, the mixture was irradiated with visible light, to potentially allow for an easier incorporation of the guest molecules in cis form into the pore channels.

\section{S1.3 Synthesis of MIL-53(Al)}


The synthetic procedure for MIL-53(Al) was modified according to the previously reported procedures $[1,2]$. Aluminium oxide nonahydrate $\left(\mathrm{Al}\left(\mathrm{NO}_{3}\right)_{3} .9 \mathrm{H}_{2} \mathrm{O}\right)(1.34 \mathrm{~g}, 3.6$ $\mathrm{mmol}$ ), and terepthalic acid ( $0.30 \mathrm{~g}, 1.8 \mathrm{mmol}$ ) were mixed in $5 \mathrm{~mL}$ of distilled water in a Teflon autoclave. The mixture was heated to $200{ }^{\circ} \mathrm{C}$ and the temperature was held for $72 \mathrm{~h}$, followed by cooling the mixture to room temperature. The off-white powder was filtered and washed thoroughly with distilled water. Purification methods (calcination and solvent activation) were carried out for the removal of unreacted and embedded terephthalic acid. Firstly, as-synthesised MIL-53(Al) samples were calcined at $330{ }^{\circ} \mathrm{C}$ for 3 days. This was then followed by sonicating the sample in DMF at $70^{\circ} \mathrm{C}$ for $1 \mathrm{~h}$. Finally, the sample was soaked in $\mathrm{MeOH}$ for 2 days. The sample was then filtered, washed with $\mathrm{MeOH}$ and dried to give a white powder. Further activation of MIL-53(Al) involved heating the sample at $200{ }^{\circ} \mathrm{C}$ at a rate of $1{ }^{\circ} \mathrm{C} / \mathrm{min}$ under vacuum overnight.

\section{S1.4 Synthesis of MIL-53(Al)@MR}

MIL-53(Al) (15 wt \%: 0.51 g, 1.4 mmol, 50 wt \%: $0.62 \mathrm{~g}, 1.6 \mathrm{mmol}$ ) and methyl red (MR) (15 wt \%: $0.19 \mathrm{~g}$, $0.7 \mathrm{mmol}$, 50 wt \%: $0.62 \mathrm{~g}, 2.3 \mathrm{mmol}$ ) were mixed in DCM and the mixture was sonicated for about 15 minutes and left overnight. The mixture was filtered and thoroughly washed with DCM to remove excess MR. The orange colored MIL-53(Al)@MR was heated under vacuum at $150{ }^{\circ} \mathrm{C}$ overnight at a rate of $1{ }^{\circ} \mathrm{C} / \mathrm{min}$ to ensure solvent removal.

\section{Section 2: Thermogravic Analyses S2}

\section{S2.1 General Thermogravic Analysis Procedure}

TGA data was obtained on a TGA Mettler Toledo TGA/SDTA 851 or Mettler Toledo TGA/DSC 1 instrument with the temperature ramped from room temperature to $700{ }^{\circ} \mathrm{C}$ at a heating rate of $5{ }^{\circ} \mathrm{C} / \mathrm{min}$ under $\mathrm{N}_{2}$ gas flow $(20 \mathrm{~mL} / \mathrm{min})$. The experiment was performed under inert atmosphere (nitrogen) to minimize possible thermal oxidative degradation in air. Ceramic pans were used as they are reusable and can withstand 
high temperature of up to 700 and $1200{ }^{\circ} \mathrm{C}$ respectively. The pans were thoroughly cleaned between experiments. Ethanol was used to clean a platinum solvent and ceramic pan was heated up to $1100{ }^{\circ} \mathrm{C}$ in a furnace for 2 hours. A $70 \mu \mathrm{L}$ alumina pan was used on Mettler instruments to contain approximately $4 \mathrm{mg}$ of the sample, which had been activated accordingly.

a

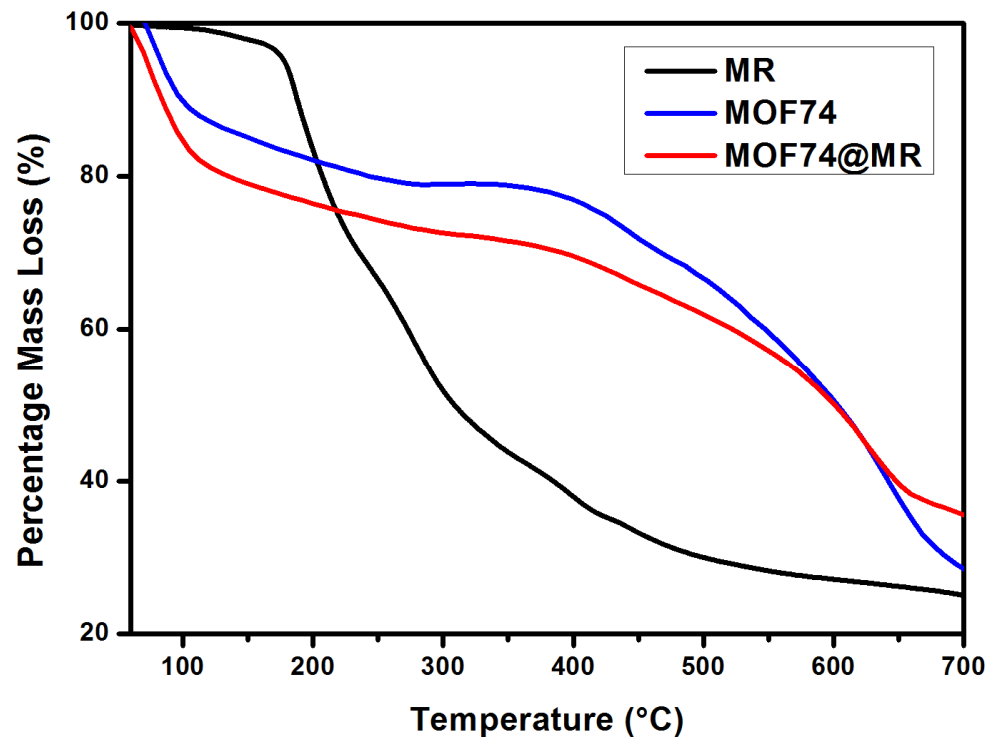

b

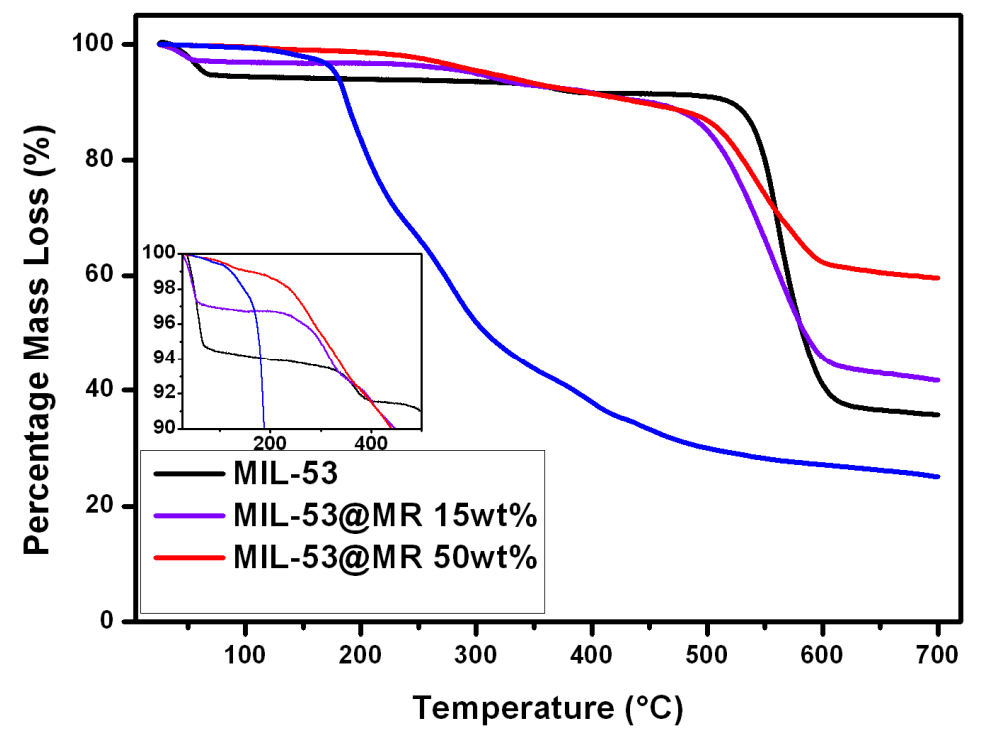

Figure SI-1. TGA curves of Mg-MOF-74@MR (a) and MIL-53(Al)@MR (b) in $\mathrm{N}_{2}$ (heating rate: $5^{\circ} \mathrm{C} / \mathrm{min}$ ). The sample was compared to the parent MOF and pure MR to confirm MOF-MR interactions. 


\section{Section 3: X-Ray Powder Diffraction S3}

S3.1 General Procedure

PXRD data were also collected on the Bruker D8 Diffractometer ( $\mathrm{Cu} K \alpha$ radiation, 40 $\mathrm{kV}, 40 \mathrm{~mA}$ ). The powder data were recorded in the range of $3.5<2 \theta<40$ with $0.020^{\circ}$ $2 \theta$ step size and 0.4 or 1 seconds/step scan speed. The activated light responsive samples were mounted on zero background plates. The activated powder samples were left to relax in the dark under vacuum overnight prior measurements in air. A black cloth was used to cover the X-ray instrument when collecting samples in the dark.

S3.1.1 Mg-MOF-74
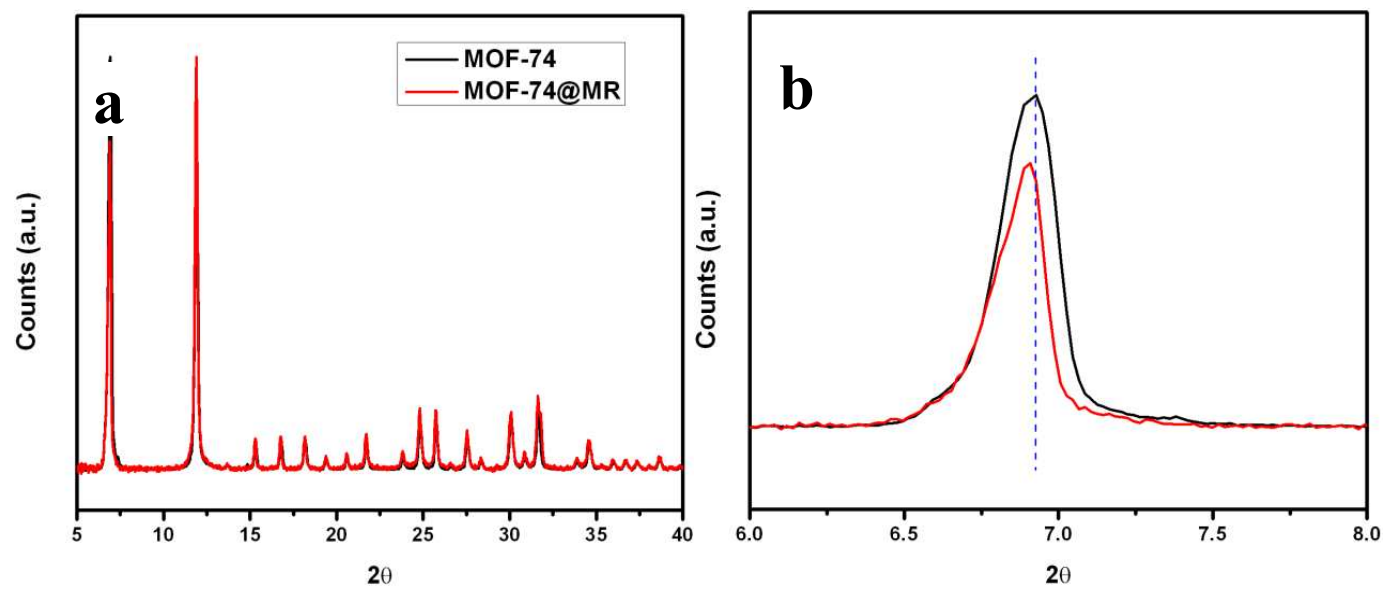

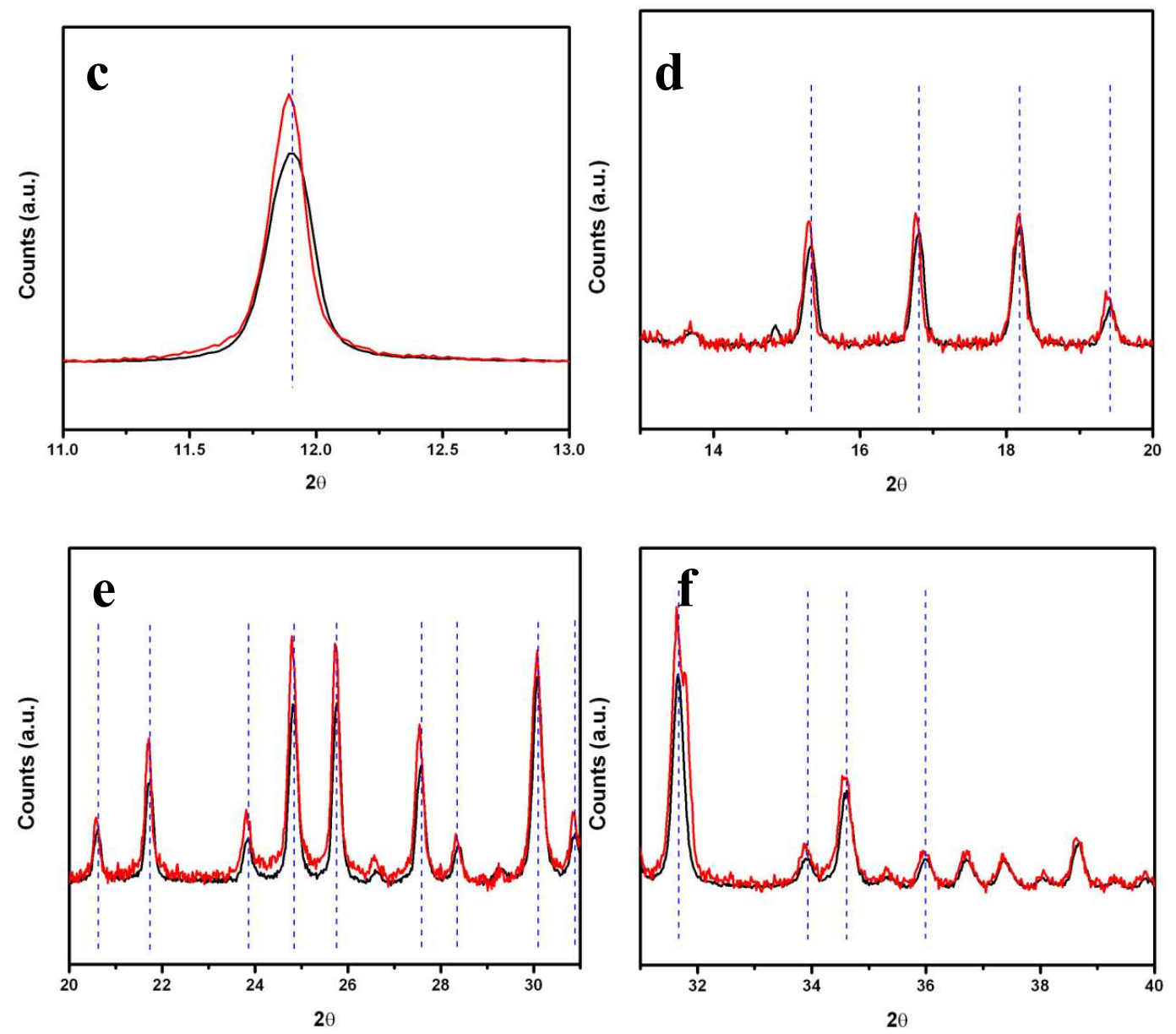

Figure SI-2. Normalised PXRD data of Mg-MOF-74 and Mg-MOF-74@MR before irradiation. An overall spectra (a) and magnified regions of the spectra (b-f) were plotted to show general peak shifts and broadening upon MR loading. 
S3.1.2 MIL-53(Al)

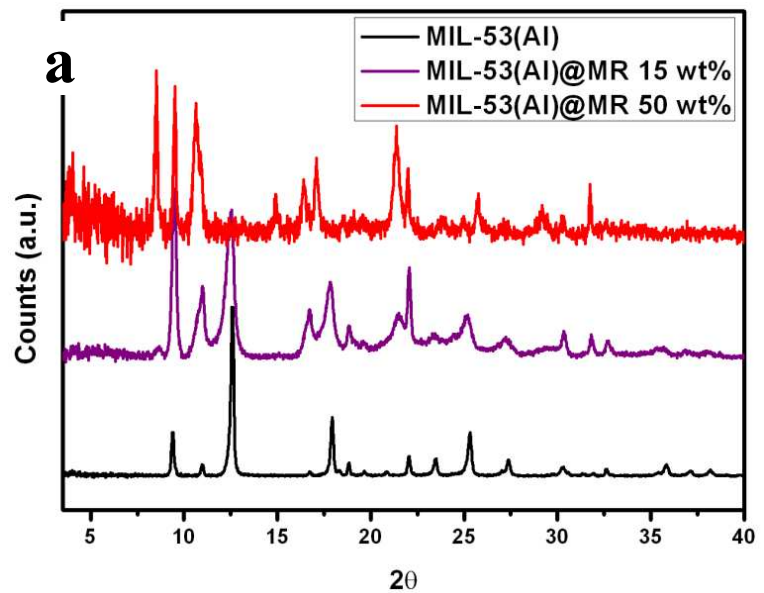



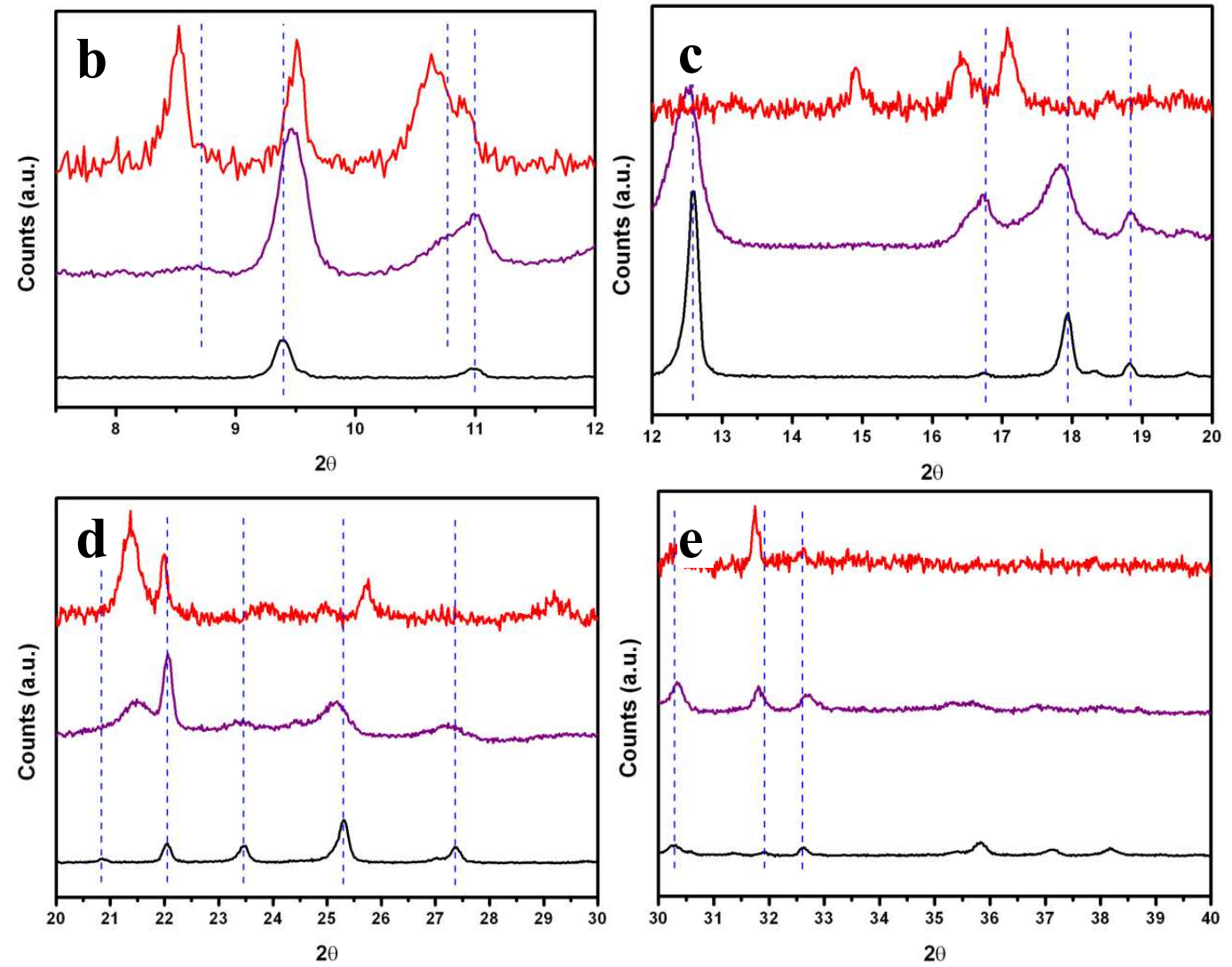

Figure SI-3. Normalised PXRD data of MIL-53(Al) and MIL-53(Al)@MR 15 wt \% and $50 \mathrm{wt} \%$ guest loading before irradiation. An overall spectra (a) and magnified regions of the spectra (b-e) were plotted to show general peak shifts and broadening with different MR loadings.

\section{Section 4: Photoresponse Characterisation $\mathrm{S}_{5}$}

All samples were used in solid state. Free ligand refers to unbound ligand in a solid state. In all UV-VIS, FTIR and PXRD experiments, Acticure 4000 light source was used to irradiate the sample. To achieve visible light irradiation, 400-500 nm filter was used with an intensity of $8,100 \mathrm{mWcm}^{-2}$.

\section{S4.1 UV-VIS Analyses}

UV-VIS spectra were obtained using Agilent Cary 6o. The solid samples used were activated accordingly prior to measurements. The spectrophotometer was used to measure the solid sample in $\mathrm{KBr}$ form. The spectrophotometer was calibrated against air and background correction was then carried out prior to measurement. For photo- 
response analyses, the sample was irradiated with light for a certain period of time and the spectra were recorded immediately after irradiation.
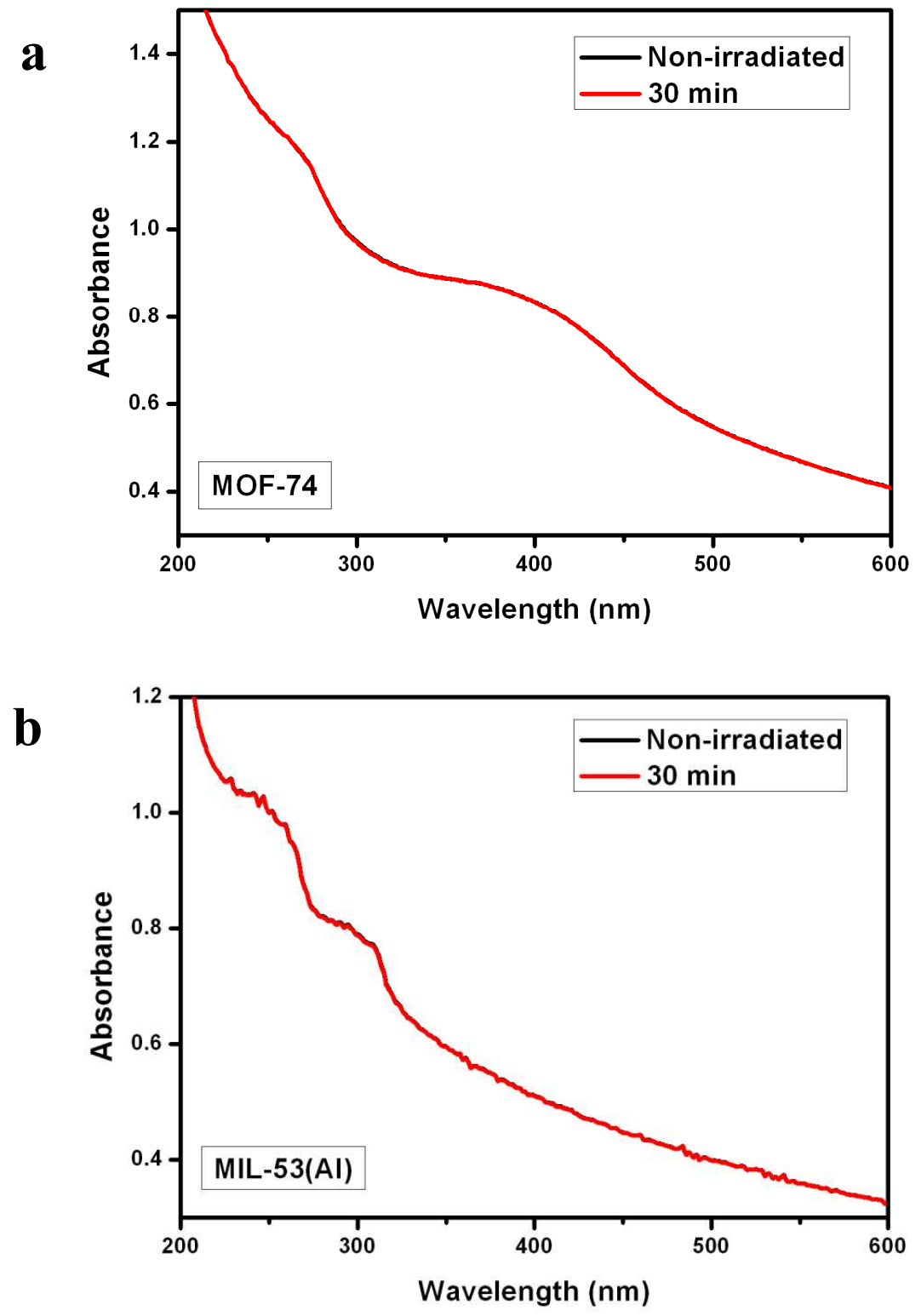

Figure SI-4. UV-VIS spectra of non-irradiated and irradiated Mg-MOF-74 (a) and MIL-53(Al) (b) in air with visible light (400-500 $\mathrm{nm})$.

S4.2. FT-IR Analyses 
Infrared spectra were obtained on Thermoscientific Nicolet 6700 in powder form with air as the background reference. For photoresponse studies, samples were measured on Agilent Cary 630 in $\mathrm{KBr}$ form.

Photo-response experiments were conducted by irradiating the solid sample for 30 minutes and the spectra were recorded immediately after irradiation. The solid samples used were activated under vacuum at $150{ }^{\circ} \mathrm{C}$ overnight at a rate of $1{ }^{\circ} \mathrm{C} / \mathrm{min}$ prior to measurements.

S4.2.1 Mg-MOF-74 

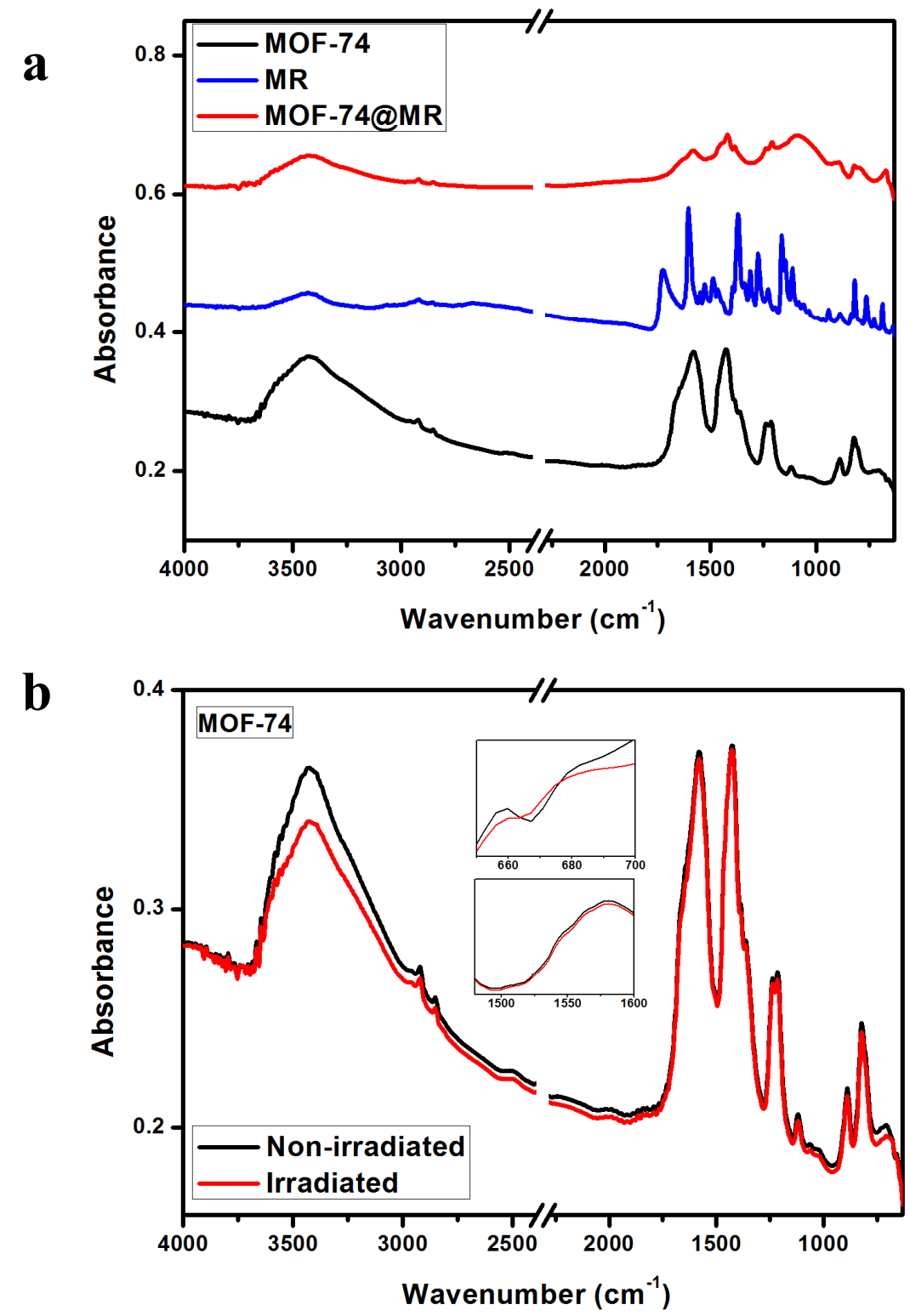

Figure SI-5. FT-IR spectra of all the materials used in the study (a), and nonirradiated and irradiated Mg-MOF-74 (b) in air with visible light (400-500 $\mathrm{nm}$ ). Peak due to $\mathrm{CO}_{2}$ in the atmosphere at $\sim 2450 \mathrm{~cm}^{-1}$ was omitted for clarity. 


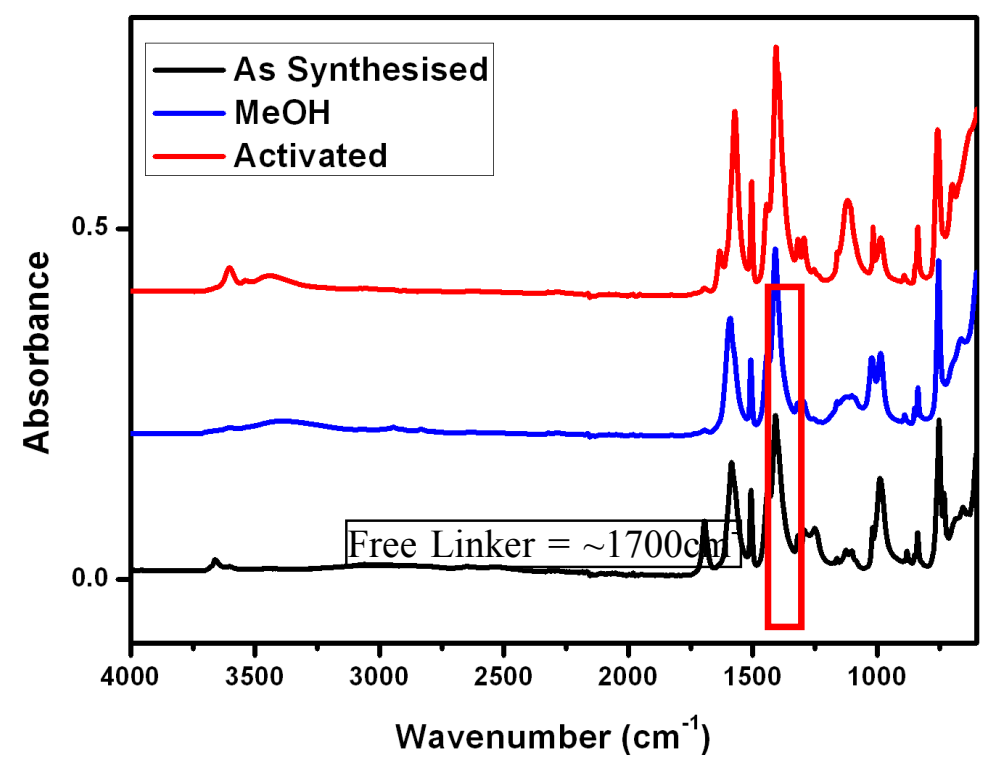

Figure SI-6. FT-IR spectra of as-synthesised, Methanol exchanged and activated MIL-53(Al) to indicate the removal of free terephthalic acid as shown by the elimination of a peak at $\sim 1690 \mathrm{~cm}^{-1}$. 
S4.2.2 $M I L-53(A l)$
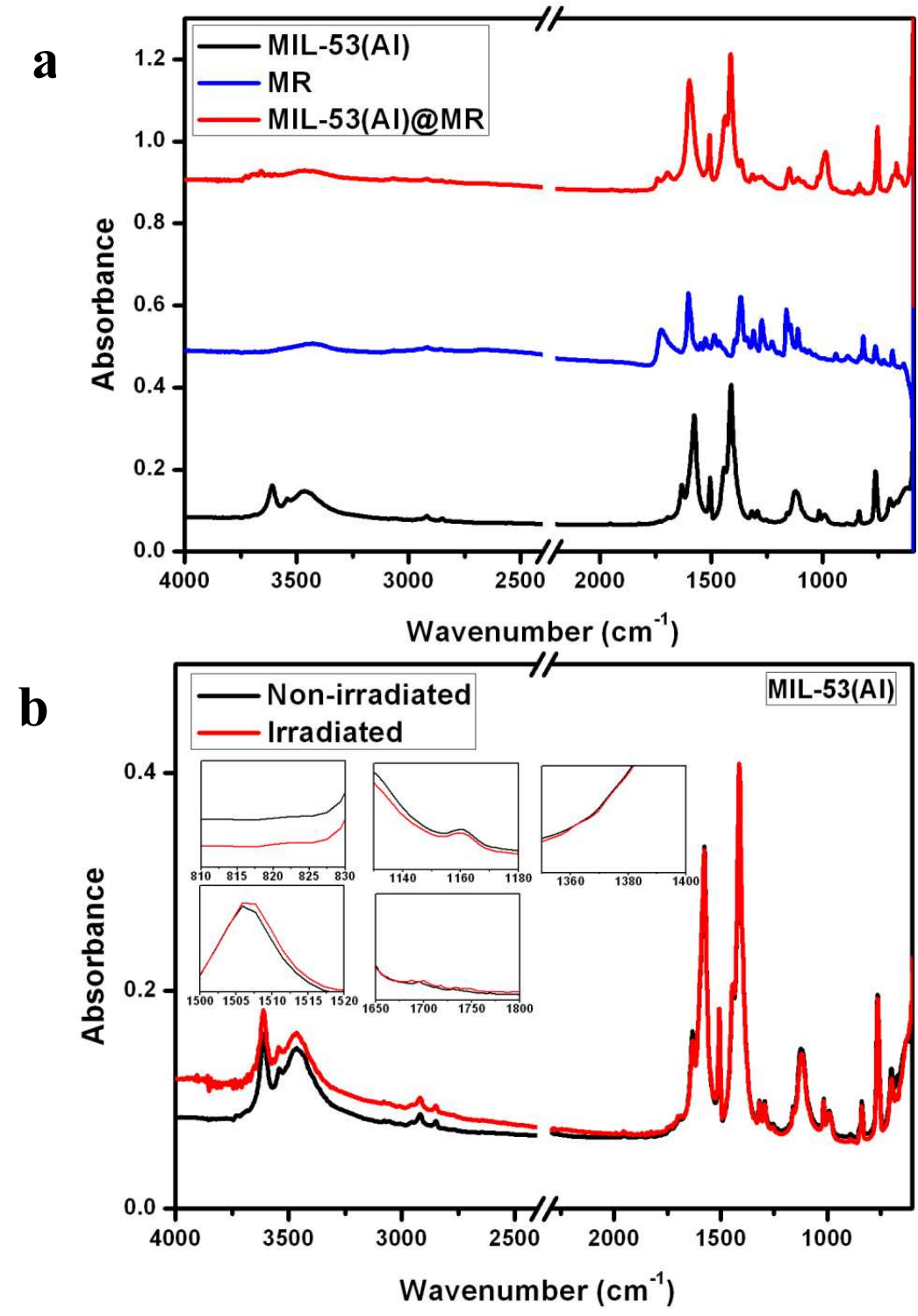

Figure SI-7. FT-IR spectra of all the materials used in the study (a), and nonirradiated and irradiated MIL-53(Al) (b) in air with visible light (400-500 nm).

Peak due to $\mathrm{CO}_{2}$ in the atmosphere at $\sim 2450 \mathrm{~cm}^{-1}$ was omitted for clarity. 
S4.4 PXRD Analyses

PXRD data were also collected on the Bruker D8 Diffractometer ( $\mathrm{Cu} \mathrm{K} \alpha$ radiation, 40 $\mathrm{kV}, 40 \mathrm{~mA}$ ). The powder data were recorded in the range of $3.5<2 \theta<40$ with $0.020^{\circ}$ $2 \theta$ step size and 0.4 or 1 seconds/step scan speed. For photoresponse experiments, activated light responsive samples were mounted on zero background plates. The activated powder samples were left to relax in the dark under vacuum overnight prior measurements in air. The experiment was set-up so that the light source was positioned over the sample in a way that shadowing of the sample did not occur. A black cloth was used to cover the X-ray instrument when collecting samples in the dark. 
S4.4.1 Mg-MOF-74
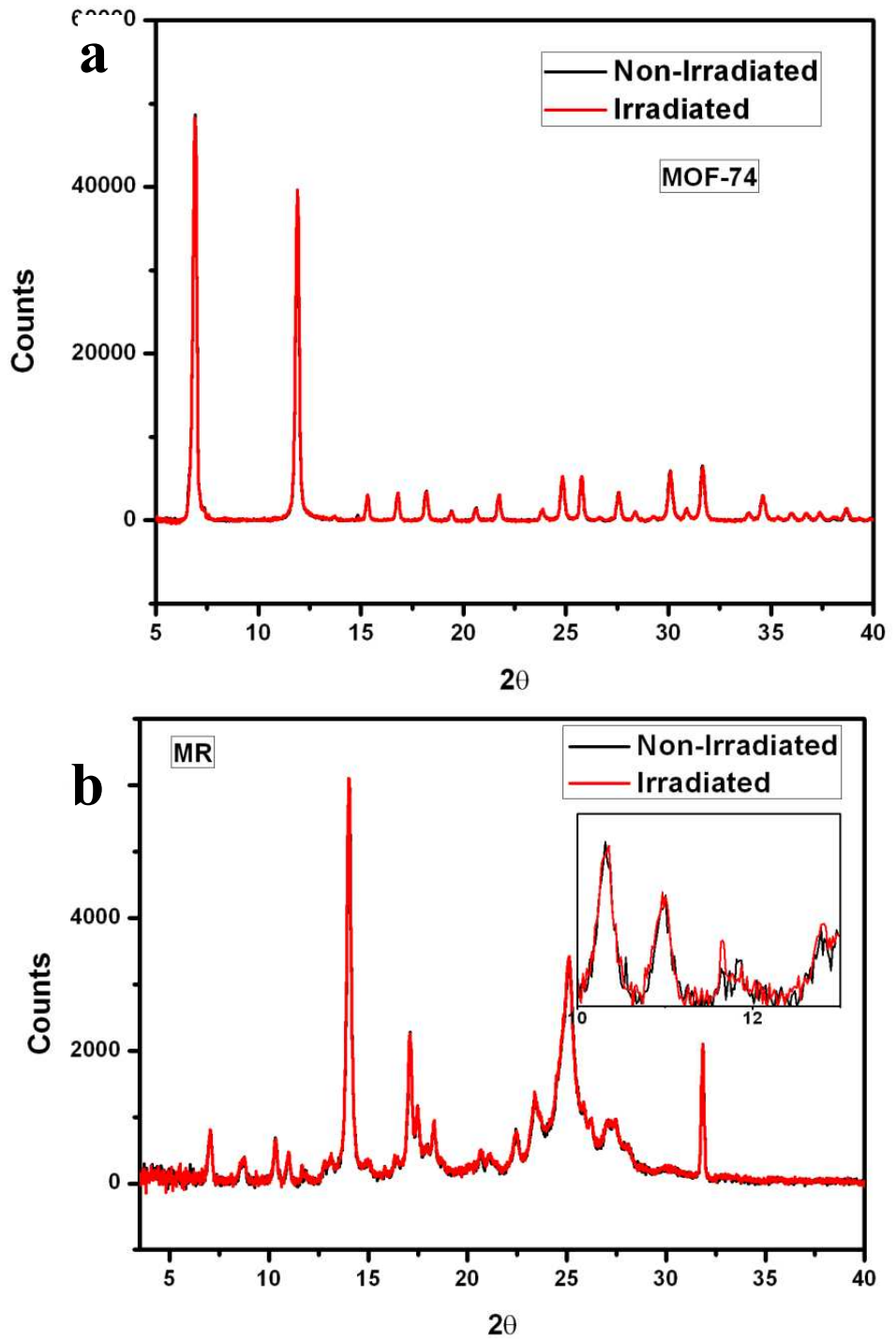

Figure SI-8. PXRD patterns of non-irradiated and irradiated Mg-MOF-74 (a) and MR (b) in air with visible light (400-500 $\mathrm{nm})$. 

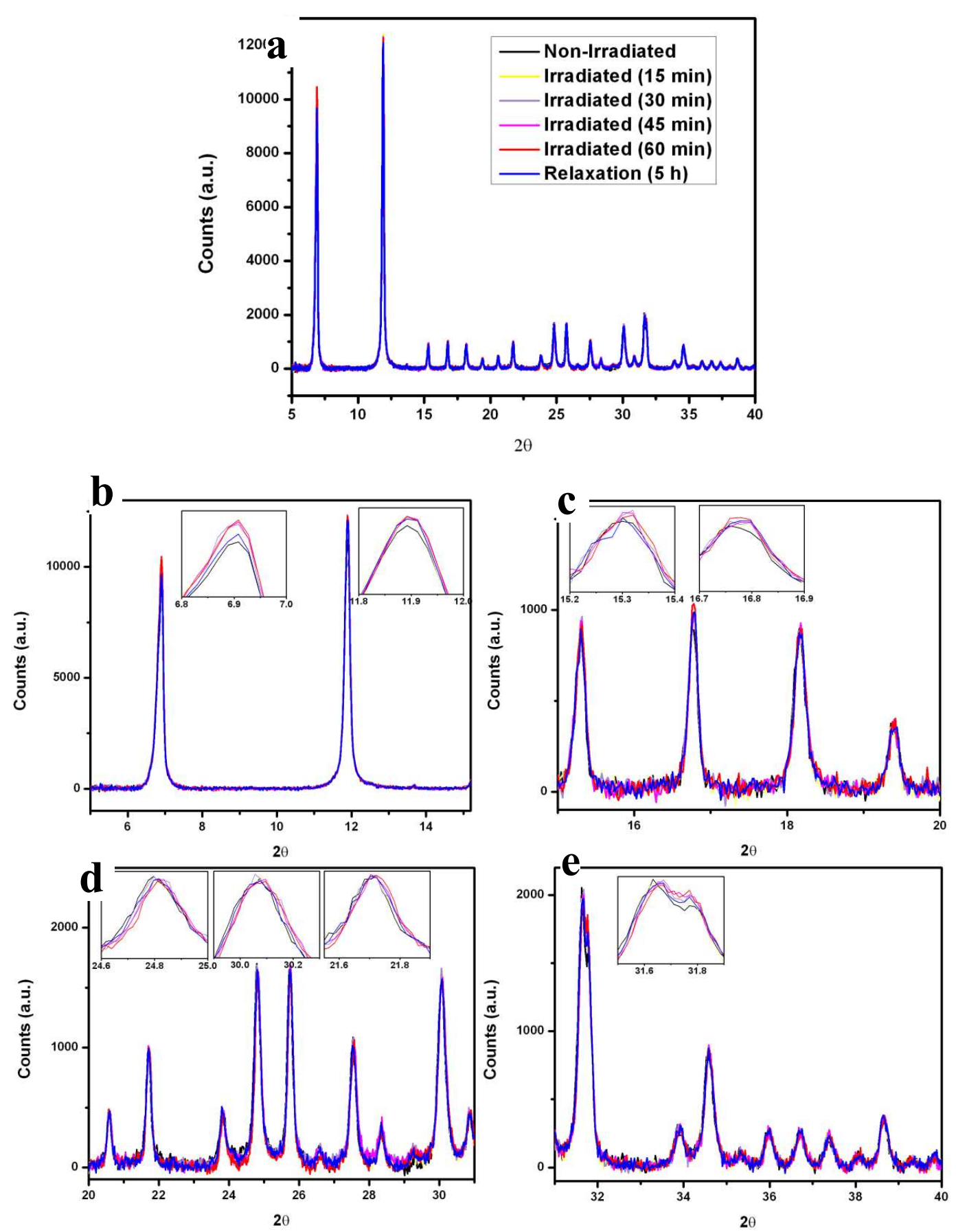

FigureSI-9. PXRD data of non-irradiated (black), irradiated (yellow, purple, magenta and red) and post-irradiated (blue) Mg-MOF-74@MR. An overall spectra (a) and magnified regions of the spectra (b-e) were plotted to show the peak changes during and after visible light irradiation (400-500 nm). PXRD pattern of the post-irradiated sample was measured 5 hours after irradiation in the dark. 
S4.4.2 MIL-53(Al)
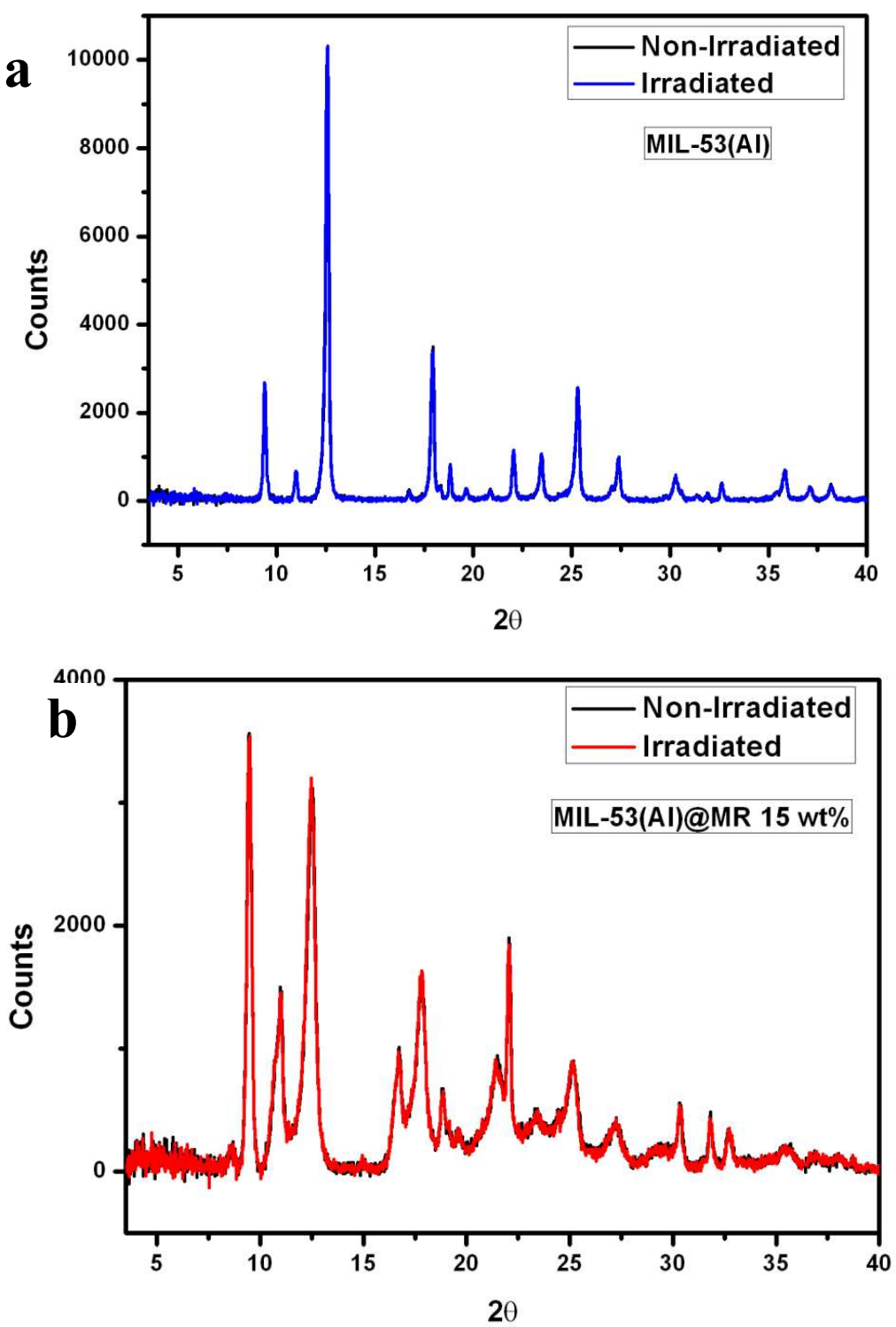


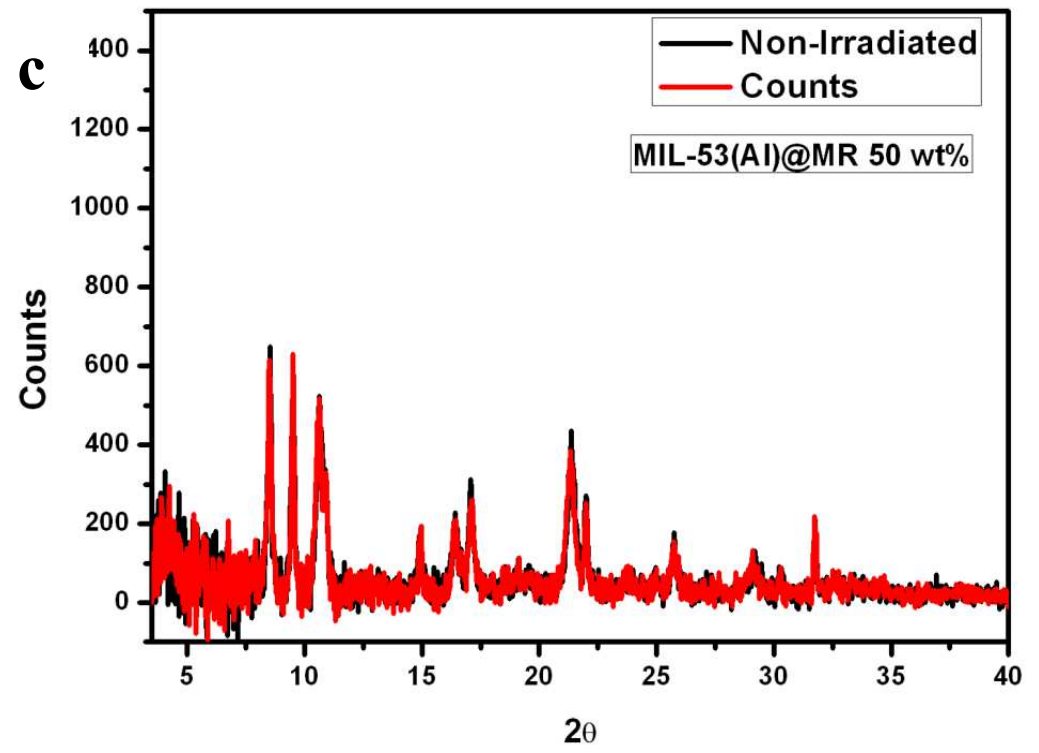

Figure SI-10. PXRD patterns of non-irradiated and irradiated MIL-53(Al) (a), MIL-53(Al)@MR 15 wt \% (b) and 50 wt \% (c) in air with visible light (400-500 nm).

S4.5 Size of Guest Molecule versus Size of Cavity

Cavity size analysis of MIL-53(Al) and Mg-MOF-74 compared with the molecular dimensions of MR has revealed that MR can not penetrate MIL-53(Al) and is unlikely to penetrate Mg-MOF-74. The molecular dimensions of free trans-MR, as shown in Fig SI11a, were calculated using the radius of gyration with atomic van der Waals diameters defined by Bondi [5]. The minimum diameter of trans-MR is $8.8 \AA$, the maximum diameter is $15.6 \AA$ and average diameter is $10.3 \AA$. These dimensions can be considered as kinetic diameters where the van der Waals potential energy is zero [6]. Using Zeo++ [7,8], the minimum window size for penetration is calculated as $6.7 \AA$ for MIL-53(Al) and $10.8 \AA$ for Mg-MOF-74, (Fig SI-11b and Fig SI-11c). Therefore MR can not adsorb within the cavities of MIL-53(Al) but could adsorbed within Mg-MOF-74 given a specific orientation. This orientation is rare considering all available degrees of freedom, and therefore MR is sterically hindered from entering Mg-MOF-74. This information supports the coating mechanism of MR upon the surface of the MOF particles. 
Note that these calculations do not consider the flexibility of MR or the frameworks. It is possible under certain conditions, such as temperature or pressure, that other configurations exist. However, the present calculations are based on the equilibrated structures and therefore are a reliable indicator for predicting adsorption and transport mechanisms.

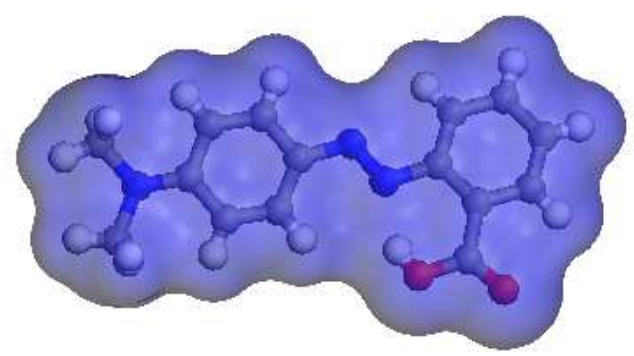

a)
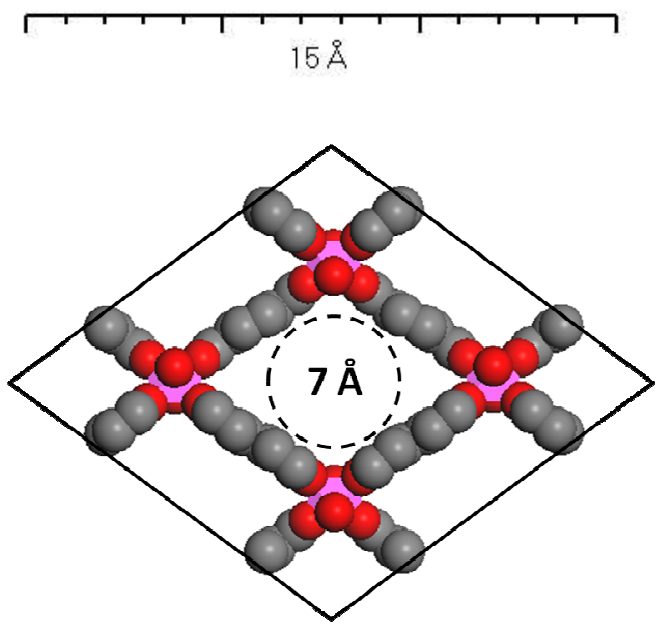

b)

c)

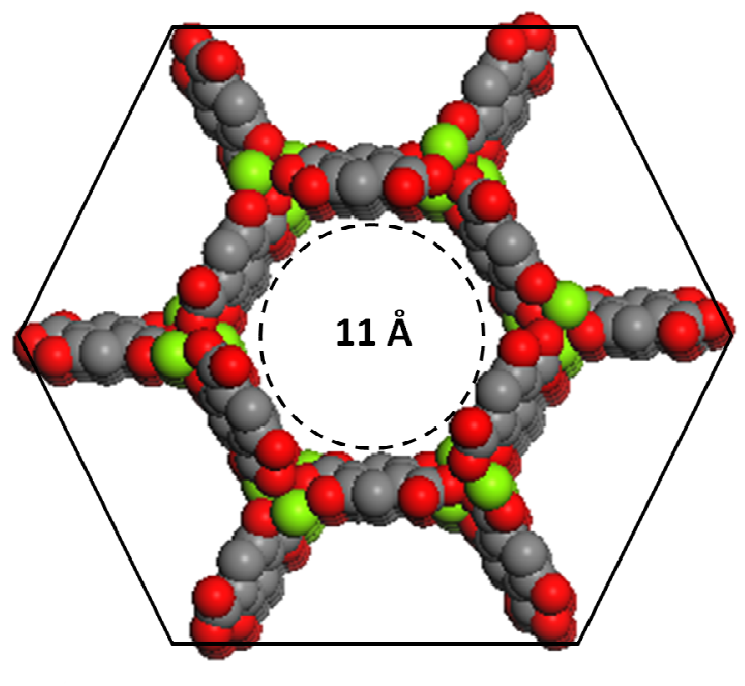




\section{Figure SI-11. Dimensions of a) free trans-MR guest molecules (average kinetic diameter: 10.3 ̊̊), b) MIL-53(Al), and c) Mg-MOF-74.}

\section{Section 5: Light-Responsive Control Experiments $\mathrm{S}_{\mathbf{5}}$}

S5.1 General Light-Responsive Control Procedure

Gas adsorption analyses were conducted on a Micromeritics Tristar using ultrahigh purity gases. During sorption experiments, 60s equilibration times were used, with less than 5\% pressure change, quite standard for these materials. Under photoswitching condition, light was switched on or off approximately every 15 minutes to allow clear detection of $\mathrm{CO}_{2}$ capture and release. No significant $\mathrm{CO}_{2}$ adsorption and release was indicated by the discontinuity of the isotherms. Photoswitching experiments were conducted on MgMOF-74 and MIL-53(Al) for a control study. Approximately $100 \mathrm{mg}$ of degassed sample was used in a glass BET tube. The samples were activated at $150{ }^{\circ} \mathrm{C}$ overnight at

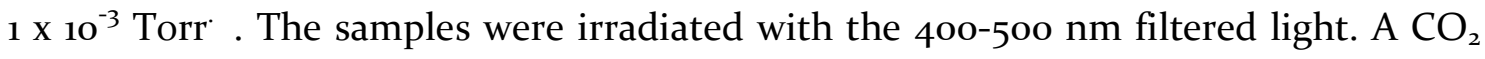
adsorption isotherm up to 1 bar was measured at $303 \mathrm{~K}$, and was consistent with the isotherms measured previously for this material without irradiation. Irradiation of MgMOF-74 and MIL-53(Al) in the absence of MR showed very low photoresponse compared to those observed in the corresponding MOF@MR as 12 \% and $0.6 \%$ reduction in uptake capacity was obtained respectively (Figure SI-10 a-b). This could be attributed to the aromatic nature of the MOF, particularly in Mg-MOF-74 where the framework itself absorbs visible light ( 400 $\mathrm{nm})$ (Figure SI-2). 

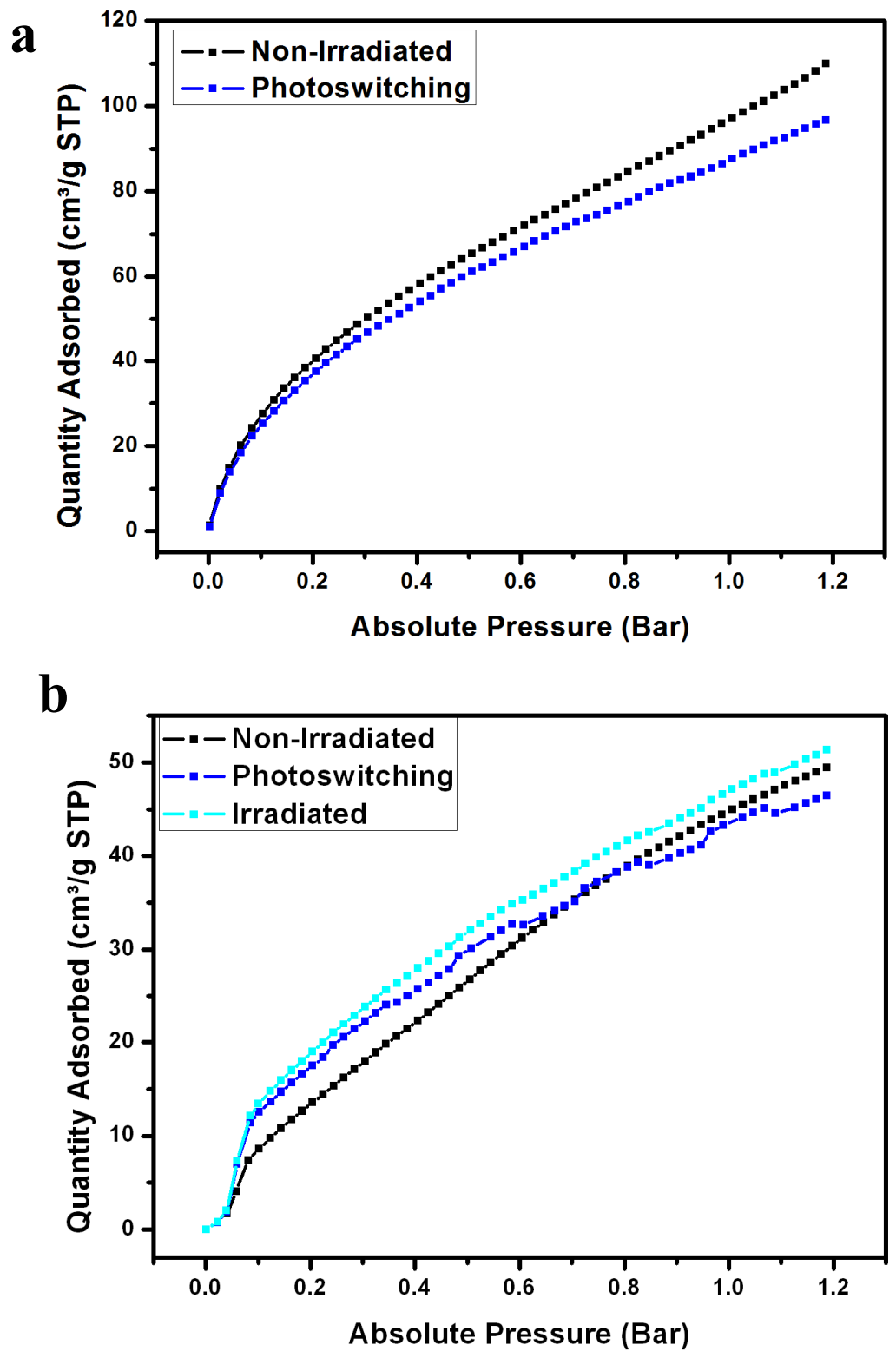

Figure SI-12. $\mathrm{CO}_{2}$ adsorption isotherm at $303 \mathrm{~K}$ of non-irradiated and irradiated Mg-MOF-74 (a) and MIL-53(Al) (b). 
S6.1 General Light-Responsive Gas Adsorption Experimental Setup

Pre-weighed and dried custom made aluminium foiled glass ASAP tube was used for light switching expiments. A custom made light cell was used to contain the ASAP tube and light guide to allow maximum light exposure and coverage on the sample when the light was switched on. A Cole Palmer Model BT 15 heated circulating bath was used to maintain the temperature at $303 \mathrm{~K}$ or $273 \mathrm{~K}$ throughout the experiment. A temperature probe was wedged inside the light cell between the ASAP tube and light guide to monitor the temperature. Acticure ${ }^{\circledR} 4000$ containing a high pressure 100 Watt mercury vapor short arc lamp was used as the light source to trigger the sample's light response during analysis. The light was fixed at the highest intensity output with 400-500 $\mathrm{nm}$ filter $\left(8,100 \mathrm{~mW} / \mathrm{cm}^{2}\right)$. The general setup are shown in the figure below.

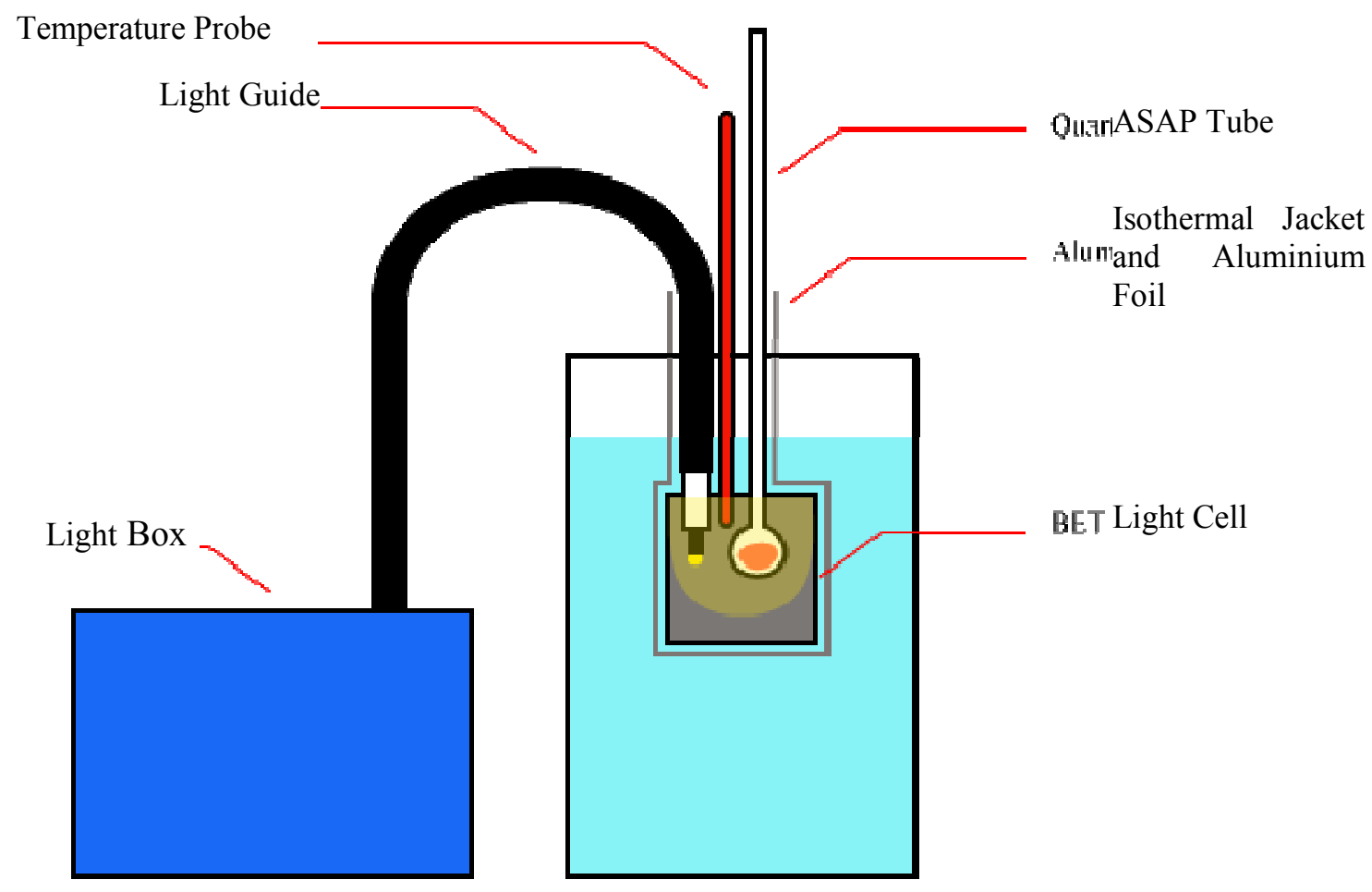

Figure SI-13. Schematic representation of the general experimental setup.

S6.2 ASAP Light Cell 
The light cell is composed of two matching halves. One half has a machined socket for a light guide and the other half has no light socket. The hemispherical cavity in each half has a smooth machined surface finish and can fit the round bottom end of an ASAP tube when clasped together. The two components are secured with four screws.
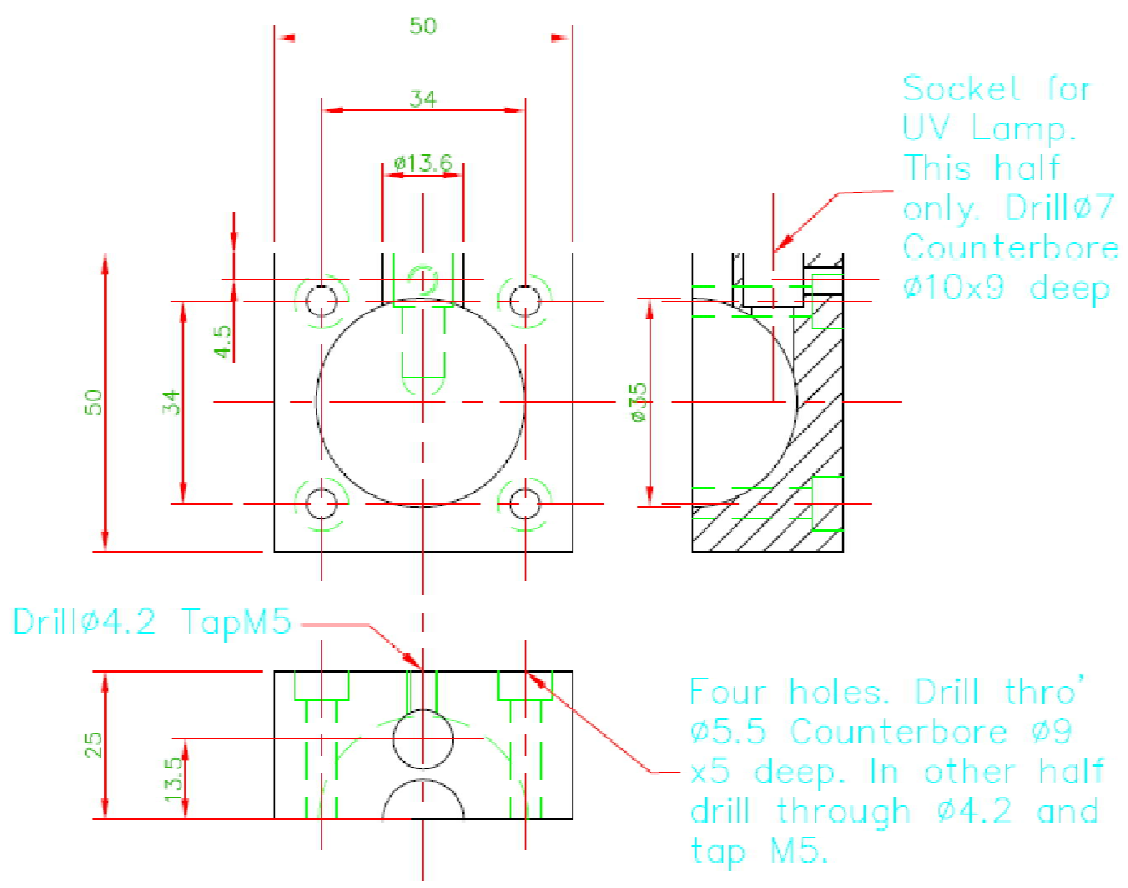

Figure SI-14. Cross section of a light cell showing a light guide socket and a smooth-surfaced cavity to allow maximum light coverage on sample. The light cell is made of stainless steel.

S6.3 Spectral Output 
The spectral outputs for visible light with $400-500 \mathrm{~nm}$ wavelength filter is shown below.

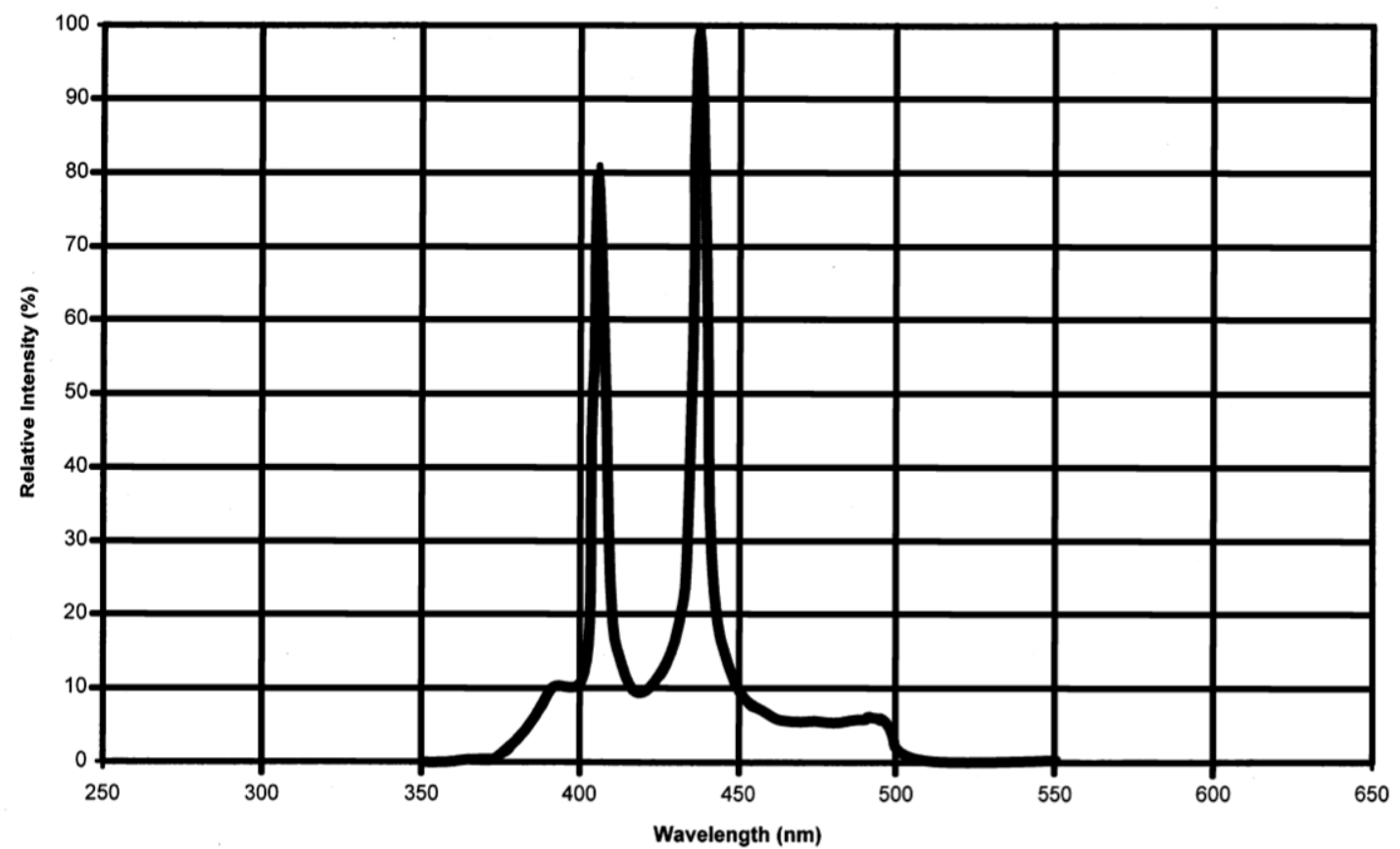

FigureSI-15. Spectral output of light using the 400-500 nm filter.

\section{References}

[1] T. Loiseau, C. Serre, C. Huguenard, G. Fink, F. Taulelle, M. Henry, T. Bataille, G. Férey, A Rationale for the Large Breathing of the Porous Aluminum Terephthalate (MIL-53) Upon Hydration. Chemistry - A European Journal, 2004, $10,1373$.

[2] P. Rallapalli, D. Patil, K. P. Prasanth, R. Somani, R. V. Jasra, H. C. Bajaj, An alternative activation method for the enhancement of methane storage capacity of nanoporous aluminium terephthalate, MIL-53(Al). J. Porous Mater., 2010, $17,523$.

[3] M. Terazima, K. Okamoto, N. Hirota, Diffusion process of methyl red in organic solvents studied by the transient grating method. J. Phys. Chem., 1993, 97, 5188.

[4] L. Xia, C. H. Wang, The holographic investigation of diffusion of methyl red in poly(caprolactone) diol. J. Chem. Phys., 1988, 88,5211 .

[5] Thornton, A. W.; Ahmed, A.; Kannam, S. K.; Todd, B. D.; Majumder, M.; Hill, A. J., Analytical Diffusion Mechanism (Adim) Model Combining Specular, Knudsen and Surface Diffusion. J. Membr. Sci. 2015, 485, 1-9.

[6] Willems, T. F.; Rycroft, C. H.; Kazi, M.; Meza, J. C.; Haranczyk, M., Algorithms and Tools for High-Throughput GeometryBased Analysis of Crystalline Porous Materials. Micropor. Mesopor. Mat. 2012, 149, 134-141.

[7] Haranczyk, M.; Rycroft, C. H.; Martin, R. L.; Willems, T. F. Zeo++: High-Throughput Analysis of Crystalline Porous Materials 0.1.0; Lawrence Berkeley National Laboratoy: Berkeley, 2012.

[8] Bondi, A., Van Der Waals Volumes and Radii. J. Phys. Chem. 1964, 68, 441-451. 
